\title{
Atypical Case of Epiphora Related to Hypoplastic Maxillary Sinus with Right Sided Deflected Nasal Septum (DNS) Masquerading as Chronic Sinusitis: Case
} Report

\author{
Shweta Sharma ${ }^{1}$, Tanu Singh ${ }^{2}$, Dhiraj Mishra ${ }^{3}$, Mir RizwanAziz ${ }^{2}$, Natasha Nargotra ${ }^{3}$, Chakshu Batra ${ }^{4}$, \\ Dashrath Rao ${ }^{4}$ and Bharat Bhushan Sharma ${ }^{5^{*}}$ \\ ${ }^{1}$ Specialty Registrar ENT, UHCW, Coventry CV 22DX UK1 \\ ${ }^{2}$ Assistant Professor, Department of Radio-diagnosis, SGT Medical College, Gurugram (Haryana), India \\ ${ }^{3}$ Senior Resident, Department of Radio-diagnosis, SGT Medical College, Gurugram (Haryana), India \\ ${ }^{4}$ PG Resident, Department of Radio-diagnosis, SGT Medical College, Gurugram (Haryana), India \\ ${ }^{5}$ Professor \& HOD, Department of Radio-diagnosis, SGT Medical College, Gurugram (Haryana), India
}

*Corresponding author: Sharma BB, Professor \& HOD, Department of Radio-diagnosis, SGT Medical College, Gurugram, 122505, India, Tel: +919811113557; E-mail: bbhushan986@gmail.com

Received: May 24, 2020; Accepted: June 07, 2020; Published: June 14, 2020

\begin{abstract}
Hypoplasia of maxillary sinus is a rare condition and that too when present on one side. This may remain asymptomatic but can present with some complaints related to overlying infective or mitotic pathologies. Sometimes it is only because of the associated complications which makes the person to report to the hospital. The diagnosis with the problems is delineated and confirmed only after the patient undergoes cross sectional imaging studies.

Case presentation: We present 11-years old male child who reported with the complaints of right nasal block, facial pain and flowing of tears from right eye of one-month duration. He had also obvious deformity of the nasal septum. These complaints were of progressive in nature which was unbearable now at the time of presentation. He underwent computerized tomography (CT) and dacrocystography (DCG) for the evaluation. The investigations confirmed the hypoplastic right maxillary sinus with superadded inflammation that had blocked the ipsilateral nasolacrimal duct. The child had been planned for dacrocysto-rhinostomy for the management as probing had failed for removing the obstruction. Conclusion: Epiphora may be related to the underlying some congenital anomaly of the sinus rather than simply maxillary sinusitis. Cross sectional imaging unveils the underlying factors and is the guide for further management.
\end{abstract}

Keywords: Hypoplasia; Nasal block; CT; DCG; Epiphora

Citation: Sharma S, Singh T, Mishra D, et al. Atypical Case of Epiphora Related to Hypoplastic Maxillary Sinus with Right Sided Deflected Nasal Septum (DNS) Masquerading as Chronic Sinusitis: Case Report. Clin Case Rep Open Access. 2020;3(2):153. 


\section{Introduction}

The incidence of maxillary sinus hypoplasia ranges from $1.4 \%$ to $10 \%$. The incidence remains asymptomatic for a long time. The common symptoms are nasal discharge, facial pain, voice difficulty and headache. The development of the maxillary sinuses starts at third fetal month and at birth this measures around $8 \mathrm{~mm}$. Then this increases in size by $2 \mathrm{~mm}$ in superoinferior and $3 \mathrm{~mm}$ in anteroposterior extent every year till 8 years of age. During the course of development, the inferior boundary of maxillary sinus and nasal cavity lie at the same level at 10 years of age. At 15 years of age the maxillary sinuses are fully developed [1].

\section{Case Report}

11-years old boy had off and on nasal block problem since his childhood. He had never shown to any otorhinolaryngologist but took some symptomatic treatment from the local village doctor (FIG. 1).

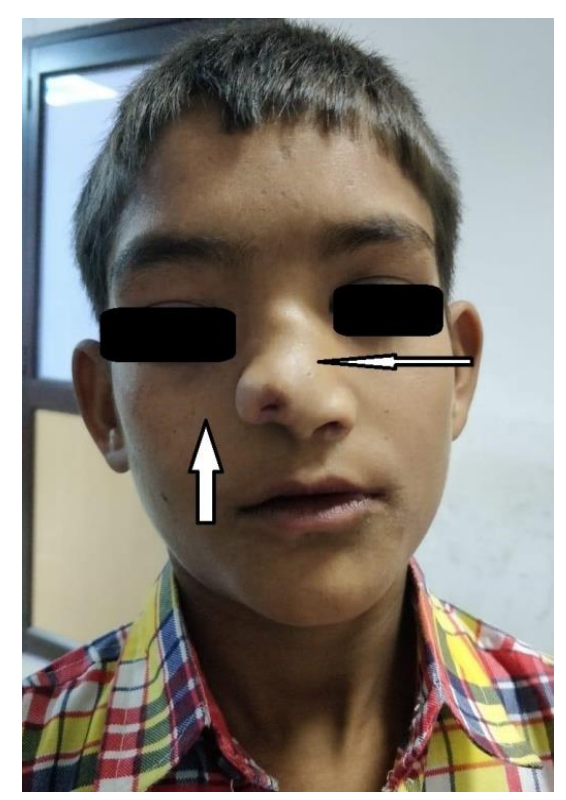

FIG.1.11-years old boy with obvious nasal deformity (horizontal white arrow) with slightly lesser right malar prominence (vertical white arrow).

Now for the last one month there was increased flow of tears in his right eye along with right nasal block, discharge and facial pain. There was mild fever for the last four days. No history of any trauma or any of the previous surgery. On examination there was obvious deformity of the nasal septum and the narrowed right nostril outlet. Temperature was $37^{\circ} \mathrm{C}$, respiratory rate 24 per minute, pulse 90 per minute with normal blood pressure readings.

Direct nasal examination had shown deviated nasal septum to right with blockade of right side nostril with some soft tissue element seen within it. Systemic examination was unremarkable. Plain X-ray (FIG. 2) could not delineate the anatomy of the sinuses well hence CT was advised for the same (FIG. 3-5). 


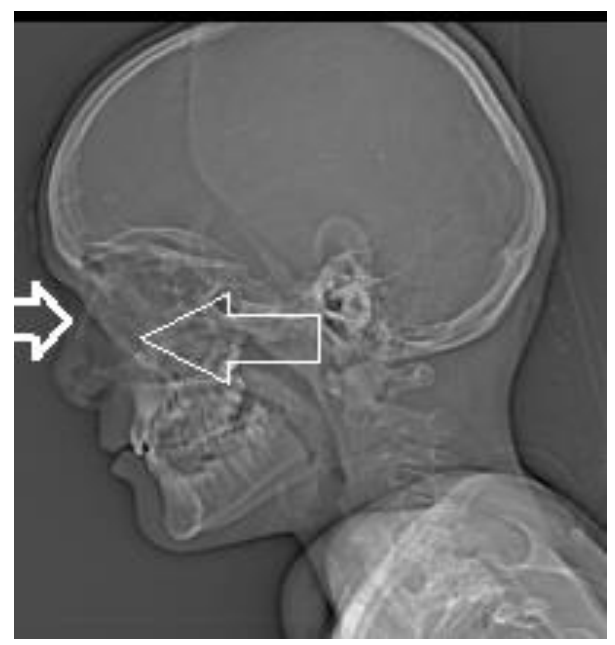

FIG 2. Plain radiograph of face and neck region in lateral profile with slight rotation to right side. The air lucency seen in naso-pharynx and oro-pharynx regions is not distorted till trachea. Nasal bone region is also well delineated

(thick white horizontal arrow). Left maxillary sinus aeration is well visualized (thin horizontal white arrow).
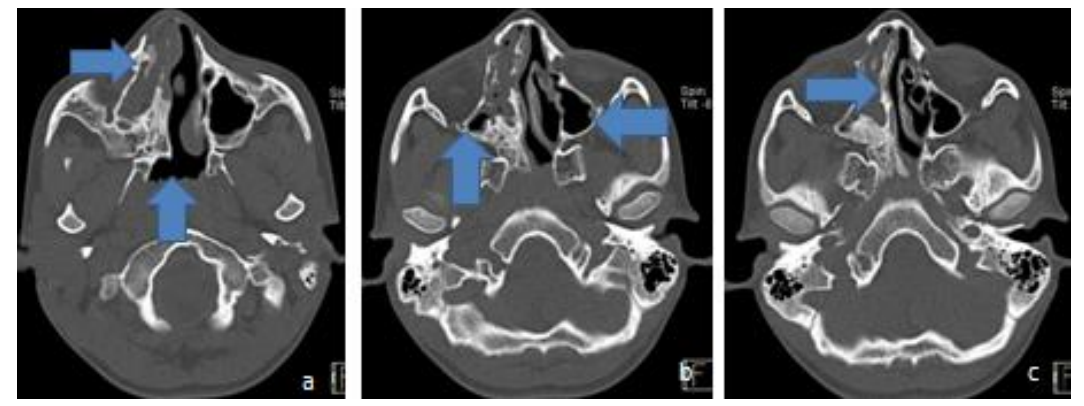

FIG 3. CT axial scans at different levels. (a) right side nasal cavity shows opacification (horizontal arrow).

Nasopharynx shows air radiolucency opposite to the posterior nares (vertical arrow). (b) Small hypoplastic distortic right maxillary sinus is seen (vertical arrow). Left maxillary sinus is aerated and delineated well (horizontal arrow).

(c) Nasal septum is deviated to right side with adjoining opacified right nasal passage (horizontal arrow).
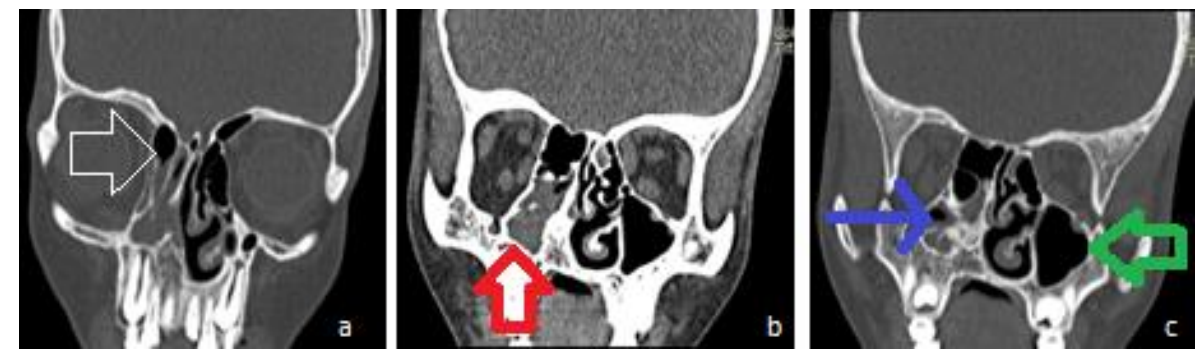

FIG 4. CT reformatted coronal sections from anterior to posterior aspect (a) anterior section which shows aerated agger nasi cells with intact lamina papyracea (white arrow). (b) section at maxillary sinus with soft tissue window shows only left maxillary sinus outline as compared to right which is distorted and hypoplastic (red vertical arrow). (c) more posterior section where a small hypoplastic right maxillary sinus is visualized (blue arrow). Left maxillary sinus outline is well defined (green horizontal hollow arrow). 

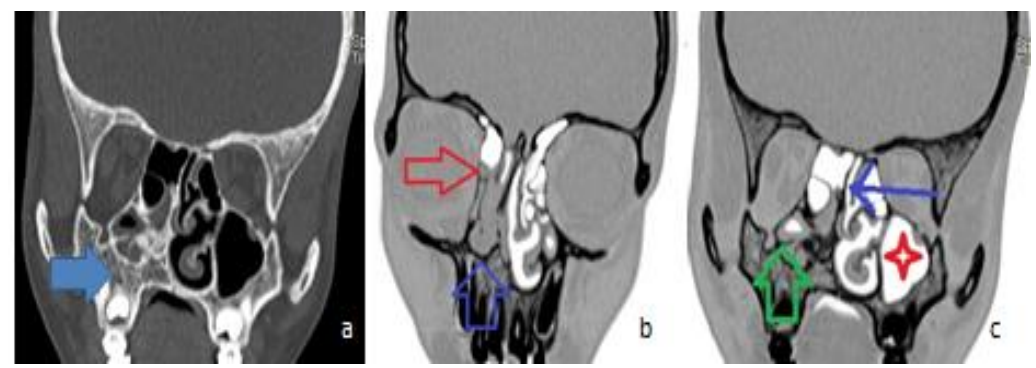

FIG 5. CT reformatted coronal section (a) distorted right maxillary sinus region (blue arrow). (b) positive image shows aerated ethmoidal cells in the upper part (red arrow) with no air in the region of right maxillary sinus in this section (blue arrow). (c) positive image in the posterior aspect shows very little air in right maxillary sinus (green arrow) as compared to left side (red star). Nasal septum is deviated to right side (blue thin arrow).

This had revealed hypoplastic right maxillary sinus with deformed outline because of underlying mucosal thickening and superadded infection. Left maxillary sinus though normal in outline but had shown the features of mucosal thickening. No air fluid level was seen. Nasal septum was deviated to right side with extensive turbinates mucosal hypertrophy causing distortion of the anatomical outlines (FIG. 6).

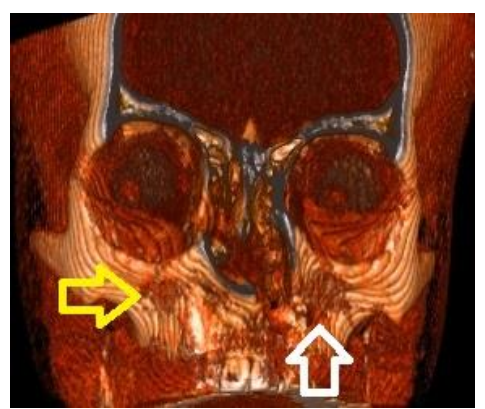

FIG 6. Volume rendered (VR) image of the face. The right maxillary sinus is hypoplastic (yellow horizontal arrow) as compared to left side (white vertical arrow).

The child was subjected to dacrocystography for the delineation of the nasolacrimal duct for evaluation of the anatomy and the obstruction site. This confirmed the obstruction and dilatation at the distal end adjacent to hypoplastic right maxillary sinus which was distorted because of inflammation (FIG. 7).

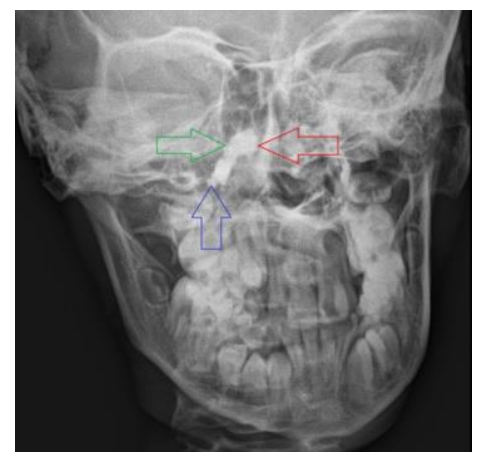

FIG 7. Dacrocystography (DCG) investigation. Antero-posterior view shows dilated nasolacrimal duct with the obstructed lower end (green hollow arrow) and proximal part (red horizontal arrow). 
www.yumedtext.com | June-2020 | ISSN: 2582-5038 | https://dx.doi.org/10.46527/2582-5038.153

The probing was done in the management after the course of antibiotics but was without any relief. The stenting was not advised because of underlying deformity of the nasal passage and ipsilateral maxillary sinus. Now the child had been planned for dacrocystorhinostomy.

\section{Discussion}

The frequency of aplasia or hypoplasia of frontal and sphenoid sinuses is common as compared to maxillary sinuses. The development of the sinus may be arrested or affected because of infection or injuries. Irradiation can also be one of the factors for hypoplasia. Congenital first arch syndrome may also be one of the hypoplastic reasons [2]. This may also be associated with the other syndromes or conditions like Down's syndrome, craniostenosis or osteodysplasia. The entity remains silent and undiagnosed for long time without any awareness to the individual. These pathologies can be misdiagnosed as simply sinusitis or some mitotic mass of the sinuses region [3]. The symptomatology is not of the same pattern in all the patients but present with different complaints at various age groups. Our present case had additional problem of deflected nasal septum to right side which further complicated the issue by epiphora. The obstruction level is classified as low, middle and lower levels. The canaliculi or Sinus of Maier is categorized as the highest level [4]. Plain X-ray may be misleading as the opacification of sinus could be due to many other reasons. Further radiological evaluation by cross sectional imaging is very important. Plain as well as contrast CT studies with 3D reconstruction clarify the underlying anatomy [5,6]. Post processing with instillation of dye in the nasolacrimal duct further adds to the diagnosis confirmation being non-invasive tool. This is very useful in pre-operative evaluation and post-operative prognostic follow-up. CT dacrocystography (CT DCG) has become very indispensible tool in the advancement of technology [7-9]. Magnetic resonance imaging may further help in tissue characterization.

In our present case DCG was required for the management of epiphora. The anatomic details are required for endoscopic surgery to avoid the damage to orbit and adjoining structures [10,11]. Dilatation, probing and flushing is the most common way of management of epiphora without any complications. Baloon catheter dilatation is another way in recurrent cases. Some patients may require stenting and intubation under general anaesthesia. Surgical intervention is by dacrocystorhinostomy either by external or endoscopic (endonasal) approach. The later is not that successful. The procedures are not without some complications related to orbit if there is gross deformity of the bone [12,13] The stents can be removed after three to six months. Nasal decongestants and eye drops are used after the surgery to prevent infections and complications.

\section{Conclusion}

Hypoplasia or aplasia of maxillary sinus is very rare as compare to other sinuses. This poses diagnostic as well as management challenge especially for the type of case we came across. The patients may not be aware of these abnormalities and report to the clinician quite late. They often report with atypical complaints like epiphora at the time of presentation as in our case. The cross sectional imaging and special investigations play a vital role in confirming the diagnosis. The management approach is decided over the correct underlying anatomy as well as pathology. 


\section{Acknowledgement}

Our sincere thanks to Mr. Nitish Kumar, Lecturer, Faculty of Allied Health Sciences and radiographers Mr. Anil Kumar, Mr. Kasif for providing the images and carrying out the desired investigations.

\section{Conflict of Interest}

There was no conflict of interest

\section{Informed and Written Consent}

The written consent was taken from the patient parents.

\section{REFERENCES}

1. Wake M, Shankar L, Hawke M, et al. Maxillary sinus hypoplasia, embryology and radiology. Arch Otolaryngol Head Neck Surg.1993;119(12):1353-7.

2. Baykara M, Erdouan N, Ozturk M. Maxillary sinus aplasia. Turk J Med Sci. 2002;32:273-5.

3. Pino RV, Pardo RG, Rodriguez CM, et al. Maxillary sinus hypoplasia masquerading a chronic sinusitis. An Otorrhinolaringol Ibero Am. 2007;34(4):323-8.

4. Pettit T, Coin C. Dacryocystography. Radiol Clin North Am. 1972;10:129-42.

5. Tasar M, Cankal F, Bozler U, et al. Bilateral maxillary sinus hypoplasia and aplasia: radiological and clinical findings. Dentomaxillofac Radiol. 2007;36(7):412-5.

6. McAlister WH, Lusk RP, Muntz HR. Comparison of plain radiographs and coronal CT scans in infants and children with recurrent sinusitis. Am J Roentgen. 1989;153(6):1259-64.

7. Munk P, Lin D, Morris D. Epiphora: treatment by means of dacyocystoplasty with balloon dilatation of the nasolacrimal drainage apparatus. Radiology.1990;177(3):687-90.

8. Khanna G, Sato Y, Smith RJ, et al. Causes of facial swelling in pediatric patients: correlation of clinical and radiologic findings. Radiographics. 2006;26(1):157-71.

9. Llyod C, McHugh K. The role of radiology in head and neck tumours in children. Can Imag. 2010;10(1):49-61.

10. Erdem T, Aktas D, Erdem G, et al. Maxillary sinus hypoplasia. Rhinology. 2002;40(3):150-3.

11. Modic MT, Weinstein MA, Berlin J, et al. Maxillary sinus hypoplasia visualized with computed tomography. Radiology. 1980;135(2):383-5.

12. Lusk RP, Muntz HR. Endoscopic sinus surgery in children with chronic sinusitis-a pilot study. Laryngoscope. 1990;100(6):654-8.

13. Stankiewicz JA. Blindness and intranasal endoscopic ethmoidectomy: prevention and management. Otolaryngol Head Neck Surg. 1989;101(3):320-9. 